Research Article

\title{
SOR-Like New Iterative Method for Solving the Epidemic Model and the Prey and Predator Problem
}

\author{
Atika Radid ${ }^{1}{ }^{1}$ and Karim Rhofir ${ }^{2}$ \\ ${ }^{1}$ University Hassan II Casablanca FSAC-MACS, BP. 5366, Maarif Casablanca, Morocco \\ ${ }^{2}$ University Sultan Moulay Slimane LaSTI-ENSA, Bd.Beni Amir, BP.77, Khouribga, Morocco \\ Correspondence should be addressed to Atika Radid; atikaradid@gmail.com
}

Received 31 March 2020; Accepted 30 June 2020; Published 18 September 2020

Academic Editor: Ewa Pawluszewicz

Copyright (C) 2020 Atika Radid and Karim Rhofir. This is an open access article distributed under the Creative Commons Attribution License, which permits unrestricted use, distribution, and reproduction in any medium, provided the original work is properly cited.

\begin{abstract}
Our aim in this paper is to propose an SOR-like new iterative method by introducing a relaxation parameter $\omega$ to improve the new iterative method proposed by Daftardar-Gejji and Jafari (NIM) [J. Math. Anal. Appl. 316 (2006) 753-763] in order to solve two problems. The first one is the problem of the spread of a nonfatal disease in a population which is assumed to have constant size over the period of the epidemic, and the other one is the problem of prey and predator. The proposed method is not limited to these two problems but can be applicable to a wide range of systems of nonlinear functional problem. The results, for different values of $\omega$, show that we found some known methods and our method compared to methods using the calculation of special polynomials and derivatives like the Adomian decomposition method (ADM), the calculation of the Lagrange multiplier as in the variational iterative method (VIM), or the construction of a homotopy as in the homotopy perturbation method (HPM) has several advantages, such as very effective and very simple to implement. Unfortunately, these methods do not guarantee a valid approximation in large time interval. To overcome this, we applied our method for approximating the solution of the problems in a sequence of time intervals as a multistage approach. Some numerical results are presented with plots according to the parameter $\omega$.
\end{abstract}

\section{Introduction}

Mathematical models are an essential tool for understanding, analysing, and describing results from natural phenomena modeling. One of the important topics studied in the field of mathematical modeling is epidemiology, because it can provide very useful results and help public health agencies better manage epidemics.

Kermack [1], a pioneer in epidemiological modeling, proposed a first epidemic model, called the SIR model, describing the spread of an infectious disease following a division of population into three distinct categories: susceptible $S$, infected $I$, and recovered $R$.

The problem of spreading of a nonfatal disease in a population which is assumed to have constant size over the period of the epidemic is considered in [2-4]. At time $t$, suppose the population consists of
$S(t)$ susceptible population: those so far uninfected and therefore liable to infection

$I(t)$ infective population: those who have the disease and are still at large

$R(t)$ isolated population, or who have recovered and are therefore immune

Assume that there is a steady constant rate between susceptible population and infective population and that a constant proportion of these constants results in transmission. Then, in time $\delta t, \delta S$ of the susceptible population becomes infective, where

$$
\delta S=-\beta S I \delta t,
$$

where $\beta$ is a positive constant. If $\gamma>0$ is the rate at which current infective population becomes isolated, then

$$
\delta I=\beta S I \delta t-\gamma I \delta t .
$$


The number of new isolated population, $\delta R$, is given by

$$
\delta R=\gamma I \delta t
$$

Now let $\delta t \longrightarrow 0$. Then, the following system determines the progress of the disease:

$$
\left\{\begin{array}{l}
\frac{\mathrm{d} S}{\mathrm{~d} t}=-\beta S(t) I(t) \\
\frac{\mathrm{d} I}{\mathrm{~d} t}=\beta S(t) I(t)-\gamma I(t), \\
\frac{\mathrm{d} R}{\mathrm{~d} t}=\gamma I(t)
\end{array}\right.
$$

with the following initial conditions:

$$
S(0)=n_{1}, I(0)=n_{2} \text {, and } R(0)=n_{3} .
$$

To solve this problem, Biazar [2, 5] used the Adomian decomposition method (ADM), Rafei et al. [3-6] used the variational iterative method (VIM) and the homotopy perturbation method (HPM), and AL-Jawary [7] used the new iterative method (NIM).

There are some rabbits and foxes living together. Foxes eat the rabbits and rabbits eat clover, and there is an increase and decrease in the number of foxes and rabbits. For more details on the mathematical modeling leading to the following system of nonlinear equations governing the problem, we refer the readers to $[6,8]$ :

$$
\left\{\begin{array}{l}
\frac{\mathrm{d} x}{\mathrm{~d} t}=x(t)(a-b y(t)), \\
\frac{\mathrm{d} y}{\mathrm{~d} t}=-y(t)(c-d x(t)),
\end{array}\right.
$$

where $x(t)$ and $y(t)$ are, respectively, the populations of the rabbits and the foxes at time $t$. To solve the prey and predator problem, Biazar et al. [2, 5] and Chowdhury et al. [9] used the Adomian decomposition and the power series methods, Rafei et al. [3] used the variational iterative method (VIM), and AL-Jawary [7] used the new iterative method (NIM).

Recently, many attempts have been made to develop analytic and approximate methods to solve the epidemic model. Although such methods have been successfully applied, some difficulties have appeared, for examples, constructing a homotopy as in the HPM $[10,11]$ and solving the corresponding the algebraic equations in calculating Adomian polynomials to handle the nonlinear terms in the ADM $[12,13]$ and calculate the Lagrange multiplier as in the VIM $[14,15]$, respectively. To overcome these difficulties, Daftardar-Gejji and Jafari [16] have proposed a new technique for solving linear/nonlinear functional equations, namely, the new iterative method (NIM) or the DJ method [17-20].

We noticed that for solving the prey and predator problem, the methods mentioned above give the accurate results but in a small time interval. Once the time interval becomes wide, these methods no longer give good results or fail. To overcome this difficulty, we introduce the multistage approach to the SOR-like new iterative method and show that our proposed approach works well when the other methods fails and gives accurate results compared to the order-four Runge-Kutta (RK4) method.

The motivation of this paper is to introduce a relaxation parameter $\omega \in(0,2)$ and propose an SOR-like new iterative method which improves the DJ method. As special cases for certain values of $\omega$, we find some wellknown methods like the new iterative method [16] and the revised new iterative method [21]. It has been used to solve effectively, easily, and accurately a large class of nonlinear problems with approximations. The main attractive features of the current method are being derivative-free, overcoming the difficulty in some existing techniques, simple to understand, and easy to implement, and the numerical results show that the approximations converge rapidly to accurate solutions.

The paper is organized as follows. Section 2 is devoted to present an SOR-like new iterative method, and for some cases for particular values of $\omega$, we find some known methods. In the case of a large interval, we present a multistage approach applied to our method. We recall a nonlinear SOR-like method [22] to solve a system of nonlinear differential algebraic equations, and we give a numerical example for comparison with our proposed method. The numerical results show that our method is more accurate, converges rapidly, and is derivative-free. In Section 3, the mathematical model of the epidemic model and the problem of the prey and predator is illustrated. The two problems are solved by the SOR-like iterative method, and the numerical results for the approximate solutions are discussed in comparison with some existing techniques, and finally in Section 4, the conclusion is presented with some possible perspectives.

\section{SOR-Like New Iterative Method}

A variety of problems in physics, chemistry, biology, and engineering can be formulated in terms of the system of nonlinear functional equations as follows:

$$
\left\{\begin{array}{l}
\frac{\mathrm{d} u_{1}}{\mathrm{~d} t}+F_{1}\left(u_{1}, \ldots, u_{i}, \ldots, u_{n}\right)=0 \\
\vdots \\
\frac{\mathrm{d} u_{i}}{d t}+F_{i}\left(u_{1}, \ldots, u_{i}, \ldots, u_{n}\right)=0 \\
\vdots \\
\frac{\mathrm{d} u_{n}}{d t}+F_{n}\left(u_{1}, \ldots, u_{i}, \ldots, u_{n}\right)=0 \\
u_{i}(0), i=1,2, \ldots, n \quad \text { initial conditions }
\end{array}\right.
$$

where $F$ is the nonlinear functional operator. Without loss of generality, one can obtain $u_{i}$ from (7) as follows: 


$$
u_{i}=f_{i}+N_{i}\left(u_{1}, u_{2}, \ldots, u_{n}\right), \quad i=1,2, \ldots, n,
$$

where $f$ is a given function and $N$ is the nonlinear operator. Equation (8) represents integral equations, ordinary differential equations (ODEs), partial differential equations (PDEs), and differential equations involving fractional order systems of ODE/PDE.

In order to solve system (8), let introduce a real parameter $\omega \in(0,2)$ and define the SOR-like new iterative method as follows.

For $k=0$,

$$
u_{i, 0}=f_{i}, \quad i=1,2, \ldots, n \text {. }
$$

For $k=1$,

$$
\left\{\begin{array}{l}
u_{1,1}=N_{1}\left(u_{1,0}, u_{2,0}, \ldots, u_{n, 0}\right), \\
u_{2,1}=N_{2}\left(\omega u_{1,1}+u_{1,0}, u_{2,0} \cdots, u_{n, 0}\right), \\
\vdots \\
u_{n, 1}=N_{n}\left(\omega u_{1,1}+u_{1,0}, \omega u_{2,1}+u_{2,0}, \ldots, \omega u_{n-1,1}+u_{n-1,0}, u_{n, 0}\right) .
\end{array}\right.
$$

For $k=2,3, \cdots$,

$$
\left\{\begin{array}{l}
u_{1, k}=N_{1}\left(\sum_{j=0}^{k-1} u_{1, j}, \ldots, \sum_{j=0}^{k-1} u_{n, j}\right)-N_{1}\left(\sum_{j=0}^{k-2} u_{1, j}, \ldots, \sum_{j=0}^{k-2} u_{n, j}\right) \\
u_{2, k}=N_{2}\left(\omega u_{1, k}+\sum_{j=0}^{k-1} u_{1, j}, \sum_{j=0}^{k-1} u_{2, j}, \ldots, \sum_{j=0}^{k-1} u_{n, j}\right)-N_{2}\left(\omega u_{1, k-1}+\sum_{j=0}^{k-2} u_{1, j}, \sum_{j=0}^{k-2} u_{2, j}, \ldots, \sum_{j=0}^{k-2} u_{n, j}\right) \\
\vdots \\
u_{n, k}=N_{n}\left(\omega u_{1, k}+\sum_{j=0}^{k-1} u_{1, j}, \ldots, \omega u_{n-1, k}+\sum_{j=0}^{k-1} u_{n-1, j}, \sum_{j=0}^{k-1} u_{n, j}\right) \\
-N_{n}\left(\omega u_{1, k-1}+\sum_{j=0}^{k-2} u_{1, j}, \ldots, \omega u_{n-1, k-1}+\sum_{j=0}^{k-2} u_{n-1, j}, \sum_{j=0}^{k-2} u_{i, j}\right) .
\end{array}\right.
$$

Suppose that system $(8)$ has a solution $u^{*}=\left(u_{1}, \ldots, u_{n}\right)$, then we are looking for $u^{*}$ having the series form:

$$
u_{i}=\sum_{j=0}^{\infty} u_{i, j}, \quad \text { for } i=1,2, \ldots, n
$$

By (10) and (11), we have for $k=1,2, \ldots$ and $i=1$

$$
\left\{\begin{array}{l}
u_{1,0}=f_{1}, \\
u_{1,1}=N_{1}\left(u_{1,0}, u_{2,0}, \ldots, u_{n, 0}\right), \\
\vdots \\
u_{1, k+1}=N_{1}\left(\sum_{j=0}^{k} u_{1, j}, \ldots, \sum_{j=0}^{k} u_{n, j}\right)-N_{1}\left(\sum_{j=0}^{k-1} u_{1, j}, \ldots, \sum_{j=0}^{k-1} u_{n, j}\right) .
\end{array}\right.
$$

Then,

$$
\begin{aligned}
& \sum_{l=0}^{k+1} u_{1, j}=f_{1}+N_{1}\left(u_{1,0}, u_{2,0}, \ldots, u_{n, 0}\right) \\
& +\sum_{l=1}^{k}\left\{N_{1}\left(\sum_{j=0}^{l} u_{1, j}, \ldots, \sum_{j=0}^{l} u_{n, j}\right)\right. \\
& \left.\quad-N_{1}\left(\sum_{j=0}^{l-1} u_{1, j}, \ldots, \sum_{j=0}^{l-1} u_{1, j}\right)\right\} \\
& =f_{1}+N_{1}\left(\sum_{j=0}^{k} u_{1, j}, \ldots, \sum_{j=0}^{k} u_{n, j}\right) .
\end{aligned}
$$

As $k \longrightarrow \infty$, we get

$$
\sum_{j=0}^{\infty} u_{1, j}=f_{1}+N_{1}\left(\sum_{j=0}^{\infty} u_{1, j}, \ldots, \sum_{j=0}^{\infty} u_{n, j}\right) .
$$

For $i=2, \ldots, n$, 


$$
\left\{\begin{array}{l}
u_{i, 0}=f_{i}, \\
u_{i, 1}=N_{i}\left(\omega u_{1,1}+u_{1,0}, u_{2,0}, \ldots, u_{n, 0}\right) \\
\vdots \\
u_{i, k+1}=N_{i}\left(\omega u_{1, k+1}+\sum_{j=0}^{k} u_{1, j}, \ldots, \omega u_{i-1, k+1}+\sum_{j=0}^{k} u_{i-1, j}, \sum_{j=0}^{k} u_{i, j}, \ldots, \sum_{j=0}^{k} u_{n, j}\right) \\
-N_{i}\left(\omega u_{1, k}+\sum_{j=0}^{k-1} u_{1, j}, \ldots, \omega u_{i-1, k}+\sum_{j=0}^{k-1} u_{i-1, j}, \sum_{j=0}^{k-1} u_{i, j}, \ldots, \sum_{j=0}^{k-1} u_{n, j}\right) .
\end{array}\right.
$$

Then,

$$
\begin{aligned}
\sum_{l=0}^{k+1} u_{i, l}= & f_{i}+N_{i}\left(u_{1,0}, u_{2,0}, \ldots, u_{n, 0}\right) \\
& +\sum_{l=1}^{k}\left\{N_{i}\left(\omega u_{1, l+1}+\sum_{j=0}^{l} u_{1, j}, \ldots, \omega u_{i-1, l+1}+\sum_{j=0}^{l} u_{i-1, j}, \sum_{j=0}^{l} u_{i, j}, \ldots, \sum_{j=0}^{l} u_{n, j}\right)\right. \\
& \left.-N_{i}\left(\omega u_{1, l}+\sum_{j=0}^{l-1} u_{1, j}, \ldots, \omega u_{i-1, l}+\sum_{j=0}^{l-1} u_{i-1, j}, \sum_{j=0}^{l-1} u_{i, j}, \ldots, \sum_{j=0}^{l-1} u_{n, j}\right)\right\} \\
= & f_{i}+N_{i}\left(\omega u_{i, k+1}+\sum_{j=0}^{k} u_{1, j}, \ldots, \omega u_{i-1, k+1}+\sum_{j=0}^{k} u_{i-1, j}, \sum_{j=0}^{k} u_{i, j}, \ldots, \sum_{j=0}^{k} u_{n, j}\right) \\
= & f_{i}+N_{i}\left(\omega \sum_{j=0}^{k+1} u_{1, j}+(1-\omega) \sum_{j=0}^{k} u_{1, j}, \ldots, \omega \sum_{j=0}^{k+1} u_{i-1, j}+(1-\omega) \sum_{j=0}^{k} u_{i-1, j}, \sum_{j=0}^{k} u_{i, j}, \ldots, \sum_{j=0}^{k} u_{n, j}\right) .
\end{aligned}
$$

As $k \longrightarrow \infty$, we get

$$
\begin{aligned}
\sum_{j=0}^{\infty} u_{i, j} & =f_{i}+N_{i}\left(\omega \sum_{j=0}^{\infty} u_{1, j}+(1-\omega) \sum_{j=0}^{\infty} u_{1, j}, \ldots, \omega \sum_{j=0}^{\infty} u_{i-1, j}\right. \\
& \left.+(1-\omega) \sum_{j=0}^{\infty} u_{i-1, j}, \sum_{j=0}^{\infty} u_{i, j}, \ldots, \sum_{j=0}^{\infty} u_{n, j}\right) \\
& =f_{i}+N_{i}\left(\sum_{j=0}^{\infty} u_{1, j}, \ldots, \sum_{j=0}^{\infty} u_{n, j}\right) .
\end{aligned}
$$

Thus, we have for $i=1,2, . ., n$,

$$
f_{i}+N_{i}\left(u^{*}\right)=f_{i}+N_{i}\left(\sum_{j=0}^{\infty} u_{i, j}, \ldots, \sum_{j=0}^{\infty} u_{i, j}\right)=\sum_{j=0}^{\infty} u_{i, j}=u_{i}^{*} .
$$

Hence, $u^{*}=\left(\sum_{j=0}^{\infty} u_{1, j}, \ldots, \sum_{j=0}^{\infty} u_{n, j}\right)$ is a solution of $(8)$.

2.1. Case 1: The New Iterative Method. We replace in (11) $\omega$ by 0 , and we find the new iterative method, also called the DG method, which is defined in $[16,20]$ by 


$$
\left\{\begin{array}{l}
u_{i, 0}=f_{i}, \\
u_{i, 1}=N_{i}\left(u_{1,0}, u_{2,0}, \ldots, u_{n, 0}\right), \\
\vdots \\
u_{i, k}=N_{i}\left(\sum_{j=0}^{k-1} u_{1, j}, \ldots, \sum_{j=0}^{k-1} u_{n, j}\right)-N_{i}\left(\sum_{j=0}^{k-2} u_{1, j}, \ldots, \sum_{j=0}^{k-2} u_{n, j}\right), \quad i=1,2, \ldots, n \text { and } k=2,3, \ldots
\end{array}\right.
$$

2.2. Case 2: The Revised New Iterative Method. We replace in (11) $\omega$ by 1 , and we find the revised new iterative method, which is defined in [21] as follows.

Initial step:

$$
u_{i, 0}=f_{i}, i=1,2, \ldots, n
$$

First iteration:

$$
\begin{aligned}
u_{1,1} & =N_{1}\left(u_{1,0}, u_{2,0}, \ldots, u_{n, 0}\right), \\
u_{2,1} & =N_{2}\left(u_{1,1}+u_{1,0}, u_{2,0} \cdots, u_{n, 0}\right), \\
& \vdots \\
u_{n, 1} & =N_{n}\left(u_{1,1}+u_{1,0}, u_{2,1}+u_{2,0}, \ldots, u_{n-1,1}+u_{n-1,0}, u_{n, 0}\right) .
\end{aligned}
$$

For $k^{\text {th }}$ iteration $(k=2,3, \cdots)$,

$$
\begin{aligned}
u_{1, k}= & N_{1}\left(\sum_{j=0}^{k-1} u_{1, j}, \ldots, \sum_{j=0}^{k-1} u_{n, j}\right)-N_{1}\left(\sum_{j=0}^{k-2} u_{1, j}, \ldots, \sum_{j=0}^{k-2} u_{n, j}\right), \\
u_{2, k}= & N_{2}\left(\sum_{j=0}^{k} u_{1, j}, \sum_{j=0}^{k-1} u_{2, j}, \ldots, \sum_{j=0}^{k-1} u_{i, j}\right) \\
& -N_{2}\left(\sum_{j=0}^{k-1} u_{1, j}, \sum_{j=0}^{k-2} u_{2, j}, \ldots, \sum_{j=0}^{k-2} u_{n, j}\right), \\
\vdots & N_{n}\left(\sum_{j=0}^{k} u_{1, j}, \ldots, \sum_{j=0}^{k} u_{n-1, j}, \sum_{j=0}^{k-1} u_{n, j}\right) \\
\left.u_{n, k}\right) & N_{n}\left(\sum_{j=0}^{k-1} u_{1, j}, \ldots, \sum_{j=0}^{k-1} u_{n-1, j}, \sum_{j=0}^{k-2} u_{i, j}\right) .
\end{aligned}
$$

2.3. Multistage SOR-Like New Iterative Method. This section is devoted to introduce the basic approach of the multistage principle: The SOR-like new iterative method as other semianalytical methods can provide valid approximate solutions only in the neighbourhood of initial time. Sometime, those methods have a slow convergence or fail when using the large domain. This drawback prevents its application to general problems. To overcome this difficulty, the multistage strategy is applied to the SOR-like new iterative method as follows:

Step 1: divide the time interval $I=[0, T]$ into $M$ subintervals, i.e., $I_{0}=\left[t_{0}, t_{1}\right], I_{1}=\left[t_{1}, t_{2}\right], \ldots, I_{M-1}=$ $\left[t_{M-1}, t_{M}\right]$ with constant step size $h=\left(t_{M}-t_{0}\right) / M$.

Step 2: at each stage, a set of differential equations will be solved using the SOR-like new iterative method with initial conditions. In order to compute approximate solutions in each stage, we need to know initial conditions at the left end point of every subinterval. Usually, we do not have these information except at the initial time $t_{0}=0$. Therefore, knowledge of a process at the initial time $t_{0}=0$ is used to calculate approximate solutions in the first subinterval $\left[t_{0}, t_{1}\right]$, and this solution serves as the initial condition for the next subinterval $\left[t_{1}, t_{2}\right]$. Likewise, we can obtain approximate solutions in every subinterval, and this procedure will continue until a desired time span is reached.

Step 3: formulation. Let $I=\cup_{j=0}^{M-1} I_{j}$ such that $I_{j}=\left[t_{j} ; t_{j+1}\right), \quad j=0, \ldots, M-1, \quad$ where $0 \leq t_{0}<t_{1}<\cdots<t_{M}=T$ and $t_{j+1}-t_{j}=h>0$. Using the initial conditions given in (7), let $u_{0}^{\star}=\left(u_{1,0}^{\star}, \ldots, u_{n, 0}^{\star}\right)$ be the solution of system $(8)$ in the subinterval $I_{0}$ given by (11).

The value at $t=t_{1}$ is used as the initial condition for the next time step. For $j=1, \ldots, M-1$, the initial condition for the next time step is written as

$$
u_{j}^{\star}\left(t_{j-1}\right)=u_{j-1}^{\star}\left(t_{j-1}\right) \text {. }
$$


Therefore, to obtain the approximated values of the $u^{\star}$ at any grid point, we define the characteristic function $\chi_{I_{j}}$ on $I_{j}$ by

$$
\begin{aligned}
& \chi_{I_{j}}(t)= \begin{cases}1, & t \in I_{j} \\
0 & t \notin I_{j}\end{cases} \\
& \forall j \in 0, \ldots, M-1 .
\end{aligned}
$$

Then, for $t \in\left[t_{0}, T\right]$, the solution $u^{\star}$ of (8) is given by $u_{i}^{\star}(t)=\sum_{j=0}^{M-1} \chi_{I_{j}}(t) u_{i, j}^{\star}(t), i=1,2, \ldots, n$ and $t \in[0, T]$.

2.4. Comparison with Nonlinear SOR-Like Method. In this section, we review the nonlinear SOR-like method defined in [22] to solve the nonlinear algebraic equations

$$
F_{i}\left(z_{1}, \ldots, z_{i}, \ldots, z_{n}\right)=0, i=1, \ldots, n,
$$

in $\mathbb{R}^{n}$ or $\mathbb{C}^{n}$, which can simply denoted by

$$
F(z)=0
$$

with $F=\left(F_{1}, \ldots, F_{n}\right)^{t}$ and $z=\left(z_{1}, \ldots, z_{n}\right)$ defined by solving the equation

$$
F_{i}\left(z_{1}^{k+1}, \ldots, z_{i-1}^{k+1}, z_{i}, z_{i+1}^{k}, \ldots, z_{i+1}^{k}\right)=0,
$$

with respect to $z_{i}$ and setting $z_{i}^{k+1}=z_{i}^{k}+w\left(z_{i}-z_{i}^{k}\right)$, $i=1,2, \ldots, n, k=0,1,2, \ldots$.

The SOR-like iteration is written in the following form:

$$
z_{i}^{k+1}=z_{i}^{k}-\omega \frac{F_{i}\left(z^{(k, i-1)}\right)}{F_{i i}\left(z^{(k, i-1)}\right)}, 1 \leq i \leq n, k \geq 0,
$$

where

$$
\begin{aligned}
F_{i i}(z)= & \frac{\partial F_{i}}{\partial z_{i}}(z), z^{(k, i-1)} \\
& =\left(z_{1}^{k+1}, \ldots, z_{i-1}^{k+1}, z_{i}^{k}, \ldots, z_{n}^{k}\right) \text { and } z^{(k, 0)} \\
& =\left(z_{1}^{k}, \ldots, z_{n}^{k}\right)=z^{(k, n)} .
\end{aligned}
$$

In order to compare this method with our proposed method, we transform the nonlinear algebraic equation (28) into the form defined in (8), by

$$
F_{i}\left(u_{1}, \ldots, u_{n}\right)=u_{i}-f_{i}-N_{i}\left(u_{1}, \ldots, u_{n}\right), \quad i=1, \ldots, n .
$$

Then, the corresponding nonlinear SOR-like method is defined by

$$
u_{i}^{k+1}=u_{i}^{k}-\omega \frac{F_{i}\left(u^{(k, i-1)}\right)}{F_{i i}\left(u^{(k, i-1)}\right)}, 1 \leq i \leq n, k \geq 0 .
$$

Example 1. Consider the following nonlinear algebraic equation:

$$
\left\{\begin{array}{l}
15 x+y^{2}-4 z-13=0 \\
x^{2}+10 y-e^{-z}-11=0 \\
y^{3}-25 z+22=0
\end{array}\right.
$$

Equation (34) is converted to

$$
\left\{\begin{array}{l}
x=\frac{13}{15}+\frac{-y^{2}+4 z}{15} \\
y=\frac{11}{10}+\frac{-x^{2}+e^{-z}}{10} \\
z=\frac{22}{25}+\frac{y^{3}}{25}
\end{array}\right.
$$

In Tables 1 and 2, we compare the number of iterations taken by both the SOR-like method (33) and the SOR-like new iterative method ((10) and (13)) for equations (34) and (35) as follows.

In Tables 1 and 2, we observe that a few terms of the proposed method (10) and (11) are enough to give an accurate solution approximation compared to the SOR-like method (33).

Remark 1. The SOR-like new iterative method is derivativefree and converges rapidly to accurate solutions. It may be observed that our method is an extension of the SOR-like method to solve a general nonlinear equation of the form (8).

One remark is that the proposed method can be considered as a relaxation of the Picard method for this type of the problem but also in the resolution of a wide class of nonlinear equations.

\section{Applications}

The existence of the solution is not easy to find for the nonlinear equations, so we use well-known approximation methods used as referenced approximation solutions.

Thus, for all numerical comparisons, the fourth-order Runge-Kutta (RK4) approximation solution will be considered as a benchmark for the considered problems. 
TABle 1: Approximate solution of equation (35) using the proposed method (33).

\begin{tabular}{|c|c|c|c|c|c|c|}
\hline Iteration & & $\omega=1.5$ & & & $\omega=0.6$ & \\
\hline$k$ & $x$ & $y$ & $z$ & $x$ & $y$ & $z$ \\
\hline 0 & 2 & 1 & -2 & 2 & 1 & -2 \\
\hline 1 & $-0,6$ & 2,204358415 & 2,962684578 & 0,96 & 1,448047366 & $-0,199128192$ \\
\hline 2 & 2, 299154229 & $-0,237343734$ & $-0,162144493$ & 0,788265842 & 1,275157468 & 0,498111281 \\
\hline 3 & 0,079931884 & 1,944118025 & 1,84195096 & 0,849963079 & 1,163177391 & 0,765014696 \\
\hline 4 & 1,618854953 & 0,308613466 & 0,400788103 & 0,928268317 & 1,101489658 & 0,866079833 \\
\hline 5 & 0,641363538 & 1,534459985 & 1,33638532 & 0,981348922 & 1,068048949 & 0,903672428 \\
\hline 6 & 1,278415615 & 0,677037115 & 0,670427726 & 1,011498015 & 1,050136643 & 0,917262819 \\
\hline 7 & 0,883125358 & 1,271218346 & 1,108043169 & 1,027249778 & 1,040716812 & 0,921957724 \\
\hline 8 & 1,140054981 & 0,868962696 & 0,80534744 & 1,035089488 & 1,035866487 & 0,923459205 \\
\hline 9 & 0,976601869 & 1,139495863 & 1,006101041 & 1,038868493 & 1,033420302 & 0,923871258 \\
\hline 10 & 1,0842944 & 0,958744191 & 0,869825588 & 1,040648498 & 1, 032209918 & 0,923943118 \\
\hline$\vdots$ & $\vdots$ & $\vdots$ & $\vdots$ & $\vdots$ & $\vdots$ & $\vdots$ \\
\hline
\end{tabular}

TABle 2: Approximate solution of equation (35) using the proposed method (10) and (11).

\begin{tabular}{lcccccc}
\hline Iteration & & $\omega=1.5$ & & \multicolumn{3}{c}{$\omega=0.6$} \\
\hline$k$ & $x$ & $y$ & $z$ & $x$ & $y$ & $z$ \\
0 & $13 / 15$ & $11 / 10$ & $22 / 25$ & $13 / 15$ & $11 / 10$ & 1.0494974 \\
1 & 1.02066667 & 1.02099108 & 0.91781927 & 1.02066667 & 0.928960301 \\
2 & 1.04192362 & 1.02915231 & 0.924121951 & 1.04095976 & 1.03282005 & 0.924928527 \\
3 & 1.04248889 & 1.03095073 & 0.923945015 & 1.04219979 & 1.0311413 & 0.92394027 \\
4 & 1.04219471 & 1.03110867 & 0.923860451 & 1.04216725 & 1.03108122 & 0.923849938 \\
5 & 1.04215045 & 1.03109521 & 0.923847799 & 1.04215142 & 1.03108949 & 0.923847506 \\
$\vdots$ & $\vdots$ & $\vdots$ & $\vdots$ & $\vdots$ & $\vdots$ \\
\hline
\end{tabular}

In order to apply the SOR-like new iterative method to solve the two systems of differential equations (4) and (6), we need to convert them into the corresponding system of the integral equation, and this can be done by applying the inverse of the operator $\mathrm{d}(.) / \mathrm{d} t$, which is the integration operator $\int_{0}^{t}(.) \mathrm{d} t$ to each equation in system (8) as follows.

3.1. The Epidemic Model. The epidemic model (4) is converted to

$$
\left\{\begin{array}{l}
S(t)=S_{0}-\beta \int_{0}^{t} S(s) I(s) \mathrm{d} s=f_{1}+N_{1}(S, I, R), \\
I(t)=I_{0}+\int_{0}^{t} \beta S(s) I(s)-\gamma I(s) \mathrm{d} s \mathrm{~d} s=f_{2}+N_{2}(S, I, R), \\
R(t)=R_{0}+\gamma \int_{0}^{t} I(s) \mathrm{d} s=f_{3}+N_{3}(S, I, R) .
\end{array}\right.
$$

where $S_{0}=n_{1}, I_{0}=n_{2}$, and $R_{0}=n_{3}$ are the initial conditions.

The $k$-term approximate solution of (4) by the SOR-like new iterative method is represented by

$$
\left\{\begin{array}{l}
S=\sum_{j=0}^{k} S_{j}, \\
I=\sum_{j=0}^{k} I_{j}, \\
R=\sum_{j=0}^{k} R_{j},
\end{array}\right.
$$

where the components $S_{j}, I_{j}$, and $R_{j}$ are recursively computed by (11) with $N$ being the integral operator.

A few first approximation terms are calculated by the SOR-like new iterative method: 


$$
\begin{aligned}
& S_{1}=\left(-1.0 n_{1} n_{2} \beta\right) t+n_{1} \\
& I_{1}=-0.5 n_{1} n_{2}^{2} \beta^{2} \omega t^{2}+\left(1.0 n_{1} n_{2} \beta-1.0 n_{2} \gamma\right) t+n_{2} \\
& R_{1}=-0.166667 n_{1} n_{2}^{2} \beta^{2} \gamma \omega^{2} t^{3}-0.166667 n_{2} \gamma\left(3.0 \gamma \omega-3.0 n_{1} \beta \omega\right) t^{2}+1.0 n_{2} \gamma t+n_{3} \text {. } \\
& S_{2}=-0.125 n_{1}^{2} n_{2}^{3} \beta^{4} \omega t^{4}-1.0 \beta\left[0.333333 n_{1} n_{2} \beta\left(1.0 n_{2} \gamma n_{1} n_{2} \beta\right)-0.166667 n_{1}^{2} n_{2}^{2} \beta^{2} \omega\right] t^{3} \\
& +1.0 \beta\left[0.5 n_{1}\left(1.0 n_{2} \gamma-1.0 n_{1} n_{2} \beta\right)+0.5 n_{1} n_{2}^{2} \beta\right] t^{2}-1.0 n_{1} n_{2} \beta t+n_{1} \text {, } \\
& I_{2}=\left(0.00892857 n_{1}^{3} n_{2}^{5} \beta^{7} \omega^{3}\right) t^{7}+\left[-\beta\left(0.0138889 n_{1}^{3} n_{2}^{4} \beta^{5} \omega^{3}+0.0486111 n_{1}^{3} n_{2}^{4} \beta^{5} \omega^{2}-0.0486111 \gamma n_{1}^{2} n_{2}^{4} \beta^{4} \omega^{2} t\right)\right]^{6} \\
& +\left[-\beta\left(-0.0833333 n_{1}^{3} n_{2}^{3} \beta^{4} \omega^{2}-0.0666667 n_{1}^{3} n_{2}^{3} \beta^{4} \omega+0.075 n_{1}^{2} n_{2}^{4} \beta^{4} \omega^{2}+0.0833333 n_{1}^{2} n_{2}^{3} \beta^{3} \gamma \omega^{2}\right.\right. \\
& \left.\left.+0.133333 n_{1}^{2} n_{2}^{3} \beta^{3} \gamma \omega-0.0666667 n_{1} n_{2}^{3} \beta^{2} \gamma^{2} \omega\right)\right] t^{5} \\
& +\left[\beta\left(-0.125 n_{1}^{3} n_{2}^{2} \beta^{3} \omega+0.0416667 n_{1}^{2} n_{2}^{3} \beta^{3} \omega^{2}+0.333333 n_{1}^{2} n_{2}^{3} \beta^{3} \omega+0.25 n_{1}^{2} n_{2}^{2} \beta^{2} \gamma \omega-0.208333 n_{1} n_{2}^{3} \beta^{2} \gamma \omega-0.125 n_{1} n_{2}^{2} \beta \gamma^{2} \omega\right)\right] t^{4} \\
& +\left[\beta \left(0.333333 n_{1} n_{2}^{2} \beta \gamma-0.333333 n_{1}^{2} n_{2}^{2} \beta^{2}+0.166667 n_{1} n_{2}^{3} \beta^{2} \omega-0.333333 n_{1}^{2} n_{2}^{2} \beta^{2} \omega+0.166667 n_{1} n_{2}^{2} \beta \gamma \omega\right.\right. \\
& \left.\left.+0.166667 n_{1} n_{2}^{2} \beta^{2} \gamma \omega\right)\right] t^{3}+\left[\gamma\left(0.5 n_{2} \gamma-0.5 n_{1} n_{2} \beta\right)\right]-\beta\left[-0.5 \beta n_{1}^{2} n_{2}+0.5 \beta n_{1} n_{2}^{2}+0.5 \gamma n_{1} n_{2}\right] t^{2}+\left(1.0 n_{1} n_{2} \beta-1.0 n_{2} \gamma\right) t+n_{2} \text {, } \\
& R_{2}=\left(0.00111607 n_{1}^{3} n_{2}^{5} \beta^{7} \gamma \omega^{4}\right) t^{8}+\left[-\gamma\left(0.00198413 n_{1}^{3} n_{2}^{4} \beta^{6} \omega^{4}+0.00694444 n_{1}^{3} n_{2}^{4} \beta^{6} \omega^{3}-0.00694444 \gamma n_{1}^{2} n_{2}^{4} \beta^{5} \omega^{3} t\right)\right] t^{7} \\
& +\left[\gamma \left(0.0138889 n_{1}^{3} n_{2}^{3} \beta^{5} \omega^{3}+0.0111111 n_{1}^{3} n_{2}^{3} \beta^{5} \omega^{2}-0.0125 n_{1}^{2} n_{2}^{4} \beta^{5} \omega^{3}-0.0138889 n_{1}^{2} n_{2}^{3} \beta^{4} \gamma \omega^{3}\right.\right. \\
& \left.\left.-0.0222222 n_{1}^{2} n_{2}^{3} \beta^{4} \gamma \omega^{2}+0.0111111 n_{1} n_{2}^{3} \beta^{3} \gamma^{2} \omega^{2}\right)\right] t^{6}+\left[\gamma \left(-0.025 n_{1}^{3} n_{2}^{2} \beta^{4} \omega^{2}+0.00833334 n_{1}^{2} n_{2}^{3} \beta^{4} \omega^{3}+0.0666667 n_{1}^{2} n_{2}^{3} \beta^{4} \omega^{2}\right.\right. \\
& \left.\left.+0.05 n_{1}^{2} n_{2}^{2} \beta^{3} \gamma \omega^{2}-0.0416667 n_{1} n_{2}^{3} \beta^{3} \gamma \omega^{2}-0.025 n_{1} n_{2}^{2} \beta^{2} \gamma^{2} \omega^{2}\right)\right] t^{5} \\
& +\left[\gamma\left(-0.0833334 n_{1}^{2} n_{2}^{2} \beta^{3} \omega^{2}-0.0833333 n_{1}^{2} n_{2}^{2} \beta^{3} \omega+0.0416666 n_{1} n_{2}^{3} \beta^{3} \omega^{2}+0.0833333 \gamma n_{1} n_{2}^{2} \beta^{2} \omega^{2}+0.0833333 \gamma n_{1} n_{2}^{2} \beta^{2} \omega\right)\right] t^{4} \\
& +\left[\gamma \left(0.166667 n_{1}^{2} n_{2} \beta^{2} \omega+0.333333 n_{1} n_{2}^{2} \beta^{2} \omega^{2}-0.333333 n_{1} n_{2}^{2} \beta^{2} \omega\right.\right. \\
& \left.\left.-0.333333 n_{1} n_{2} \beta \gamma \omega+0.166667 n_{2} \gamma^{2} \omega-0.166667 n_{1} n_{2}^{2} \beta^{2} \gamma \omega^{2}\right)\right] t^{3} \\
& +\left[-\gamma\left(0.5 n_{2} \gamma-0.5 n_{1} n_{2} \beta-0.5 n_{2} \gamma \omega+0.5 n_{1} n_{2} \beta \omega-0.166667 n_{2} \gamma 3.0 \gamma \omega-3.0 n_{1} \beta \omega\right)\right] t^{2}+\left(1.0 n_{2} \gamma\right) t+n_{3} \text {. }
\end{aligned}
$$

Continuing in this manner, we can find the rest of the components which are calculated but not listed.

3.1.1. Numerical Results and Comparison with the ADM, $H P M, N I M$, and RK4. For comparison with the results obtained by Biazar [2] using the ADM, the results obtained by Rafei et al. [3], and the results obtained by AL-Jawary [7] using NIM, the following values, for parameters, are considered:

$$
\begin{cases}n_{1}=20 & \text { Initial population of } S(t), \text { who are susceptible } \\ n_{2}=15 & \text { Initial population of } I(t), \text { who are infective } \\ n_{3}=10 & \text { Initial population of } R(t), \text { who are immune } \\ \beta=0.01 & \text { Rate of change of susceptible population to infective population } \\ \gamma=0.02 & \text { Rate of change of infective population to immune population. }\end{cases}
$$

Three-, four-, five-, and six-term approximations for $S(t), I(t)$, and $R(t)$ are calculated and are plotted in Figure 1. As the plots show while the number of susceptible population increases, the population who are infective decreases in the period of the epidemic, and meanwhile the number of immune population increases. But the size of the population over the period of the epidemic is constant.
A comparison between the results obtained by the SORlike new iterative method and the NIM, ADM, and VIM in $[2,3,7]$ shows that the results of four-term approximations of the of both SOR-like and the NIM and VIM is the same as the results of six-term approximations of the ADM. In fact, the results obtained by the SOR-Like new iterative method are completely the same with the VIM and NIM, but it 
appears the results obtained by ADM in [2] are not accurate enough. Other results from comparison with order-four Runge-Kutta (RK4) and the SOR-like new iterative method for different values of the parameter $\omega$ are given in Figure 2. We notice that these plots show that our method gives better results and approaches the solution better.

3.2. The Prey and Predator Problem. The prey and predator problem is converted to

$$
\left\{\begin{array}{l}
x(t)=x_{0}+\int_{0}^{t} x(s)(a-b y(s)) \mathrm{d} s=x_{0}+N_{1}(x(t), y(t)), \\
y(t)=y_{0}-\int_{0}^{t} y(s)(c-\mathrm{d} x(s)) \mathrm{d} s=y_{0}+N_{2}(x(t), y(t)) .
\end{array}\right.
$$

By starting with initial approximations $x_{0}=x_{0}$ and $y_{0}=y_{0}$, the $k$-term approximate solution of (6) by the SORlike new iterative method is represented by

$$
\left\{\begin{array}{l}
x=\sum_{j=0}^{k} x_{j}, \\
y=\sum_{j=0}^{k} y_{j} .
\end{array}\right.
$$

A few first term approximations can be calculated by the SOR-like method:

$$
\begin{aligned}
& x_{1}=\left(x_{0} a-1.0 x_{0} y_{0} b\right) t+x_{1} \text {, } \\
& y_{1}=\left(0.5 x_{0} y_{0} a d \omega-0.5 x_{0} y_{0}^{2} b d \omega\right) t^{2}+\left(1.0 x_{0} y_{0} d-1.0 y_{0} c\right) t+y_{0} \text {. } \\
& x_{2}=\left(-0.125 d \omega x_{0}^{2} y_{0}^{3} b^{3}+0.25 d \omega x_{0}^{2} y_{0}^{2} a b^{2}-0.125 d \omega x_{0}^{2} y_{0} a^{2} b\right) t^{4}+ \\
& \left(\begin{array}{c}
0.333333 x_{0}^{2} y_{0}^{2} b^{2} d-0.333333 x_{0} y_{0}^{2} b^{2} c+0.333333 x_{0} y_{0} a b c+ \\
0.166667 x_{0}^{2} y_{0}^{2} b^{2} d \omega-0.333333 x_{0}^{2} y_{0} a b \quad d-0.166667 x_{0}^{2} y_{0} a b \quad d \omega
\end{array}\right) t^{3}+ \\
& \left(-0.5 d x_{0}^{2} y_{0} b+0.5 x_{0} y_{0}^{2} b^{2}-1.0 x_{0} y_{0} a b+0.5 c x_{0} y_{0} b+0.5 x_{0} a^{2}\right) t^{2}+ \\
& \left(x_{0} a-1.0 x_{0} y_{0} b\right) t+x_{0} \text {, } \\
& y_{2}=\left(\begin{array}{l}
0.00892857 x_{0}^{3} y_{0}^{5} b 4 d^{3} \omega^{3}-0.0267857 x_{0}^{3} y_{0}^{4} a b^{3} d^{3} \omega^{3}+ \\
0.0267857 x_{0}^{3} y_{0}^{3} a^{2} b^{2} d^{3} \omega^{3}-0.00892857 x_{0}^{3} y_{0}^{2} a^{3} b d^{3} \omega^{3}
\end{array}\right) t^{7}+ \\
& \left(-\left(\begin{array}{c}
0.0138889 x_{0}^{3} y_{0}^{2} a^{2} b d^{3} \omega^{3}-0.0486111 x_{0}^{3} y_{0}^{2} a^{2} b d^{3} \omega^{2}+0.0486111 c x_{0}^{2} y_{0}^{4} b^{3} d^{2} \omega^{2}-0.0972222 c x_{0}^{2} y_{0}^{3} a b^{2} d^{2} \omega^{2}+ \\
0.0486111 c x_{0}^{2} y_{0}^{2} a^{2} b d^{2} \omega^{2}
\end{array}\right) t^{6}\right. \\
& +\left(\begin{array}{c}
0.0833333 x_{0}^{3} y_{0}^{3} b^{2} d^{3} \omega^{2}+0.0666667 x_{0}^{3} y_{0}^{3} b^{2} d^{3} \omega-0.0833333 x_{0}^{3} y_{0}^{2} a b d^{3} \omega^{2} \\
-0.0666667 x_{0}^{3} y_{0}^{2} a b d^{3} \omega-0.075 x_{0}^{2} y_{0}^{4} b^{3} d^{2} \omega^{2}+0.2 x_{0}^{2} y_{0}^{3} a b^{2} d^{2} \omega^{2}- \\
0.0833333 x_{0}^{2} y_{0}^{3} b^{2} c d^{2} \omega^{2}-0.133333 x_{0}^{2} y_{0}^{3} b^{2} c d^{2} \omega \\
-0.175 x_{0}^{2} y_{0}^{2} a^{2} b d^{2} \omega^{2}+0.0833333 x_{0}^{2} y_{0}^{2} a b c d^{2} \omega^{2} \\
0.133333 x_{0}^{2} y_{0}^{2} a b c d^{2} \omega+0.05 x_{0}^{2} y_{0} a^{3} d^{2} \omega^{2}+0.0666667 x_{0} y_{0}^{3} b^{2} c^{2} d \omega-0.0666667 x_{0} y_{0}^{2} a b c^{2} d \omega
\end{array}\right) t^{5} \\
& +\left(\begin{array}{c}
-0.125 x_{0}^{3} y_{0}^{2} b d^{3} \omega+0.0416667 x_{0}^{2} y_{0}^{3} b^{2} d^{2} \omega^{2}+0.333333 x_{0}^{2} y_{0}^{3} b^{2} d^{2} \omega-0.0416667 x_{0}^{2} y_{0}^{2} a b d^{2} \omega^{2} \\
-0.583333 x_{0}^{2} y_{0}^{2} a b d^{2} \omega+0.25 x_{0}^{2} y_{0}^{2} b c d^{2} \omega+0.25 x_{0}^{2} y_{0} a^{2} d^{2} \omega-0.208333 x_{0} y_{0}^{3} b^{2} c d \omega \\
+0.333333 x_{0} y_{0}^{2} a b c \quad d \omega-0.125 x_{0} y_{0}^{2} b c^{2} d \omega-0.125 x_{0} y_{0} a^{2} c d \omega
\end{array}\right) t^{4}
\end{aligned}
$$

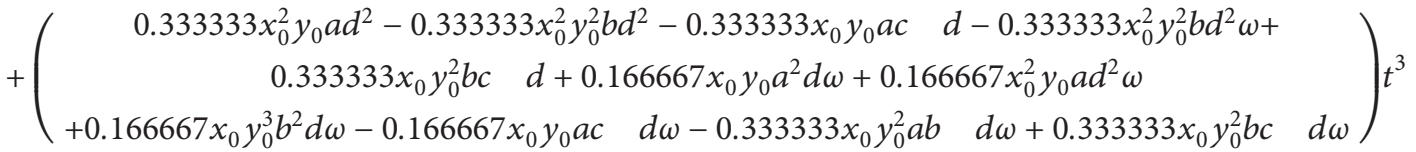

$$
\begin{aligned}
& +\left(0.5 x_{0}^{2} y_{0} d^{2}-0.5 b x_{0} y_{0}^{2} d-1.0 x_{0} y_{0} c \quad d+0.5 a x_{0} y_{0} d+0.5 y_{0} c^{2}\right) t^{2}+ \\
& \left(1.0 x_{0} y_{0} d-1.0 y_{0} c\right) t+y_{0} \text {. }
\end{aligned}
$$

Continuing in this manner, we can find the rest of the components which are calculated but not listed.

3.2.1. Numerical Results and Comparison with the ADM, $H P M, N I M$, and RK4. For comparison with the results obtained by Biazar [2] using the ADM, the results obtained by
Rafei et al. [3], and the results obtained by AL-Jawary [7] using the NIM, the following values, for parameters, are considered:

\begin{tabular}{lcccccc}
\hline Case & $x_{0}$ & $y_{0}$ & $a$ & $b$ & $c$ & $d$ \\
\hline 1 & 14 & 18 & 0.1 & 1 & 1 & 1 \\
2 & 16 & 10 & 1 & 1 & 0.1 & 1 \\
\hline
\end{tabular}



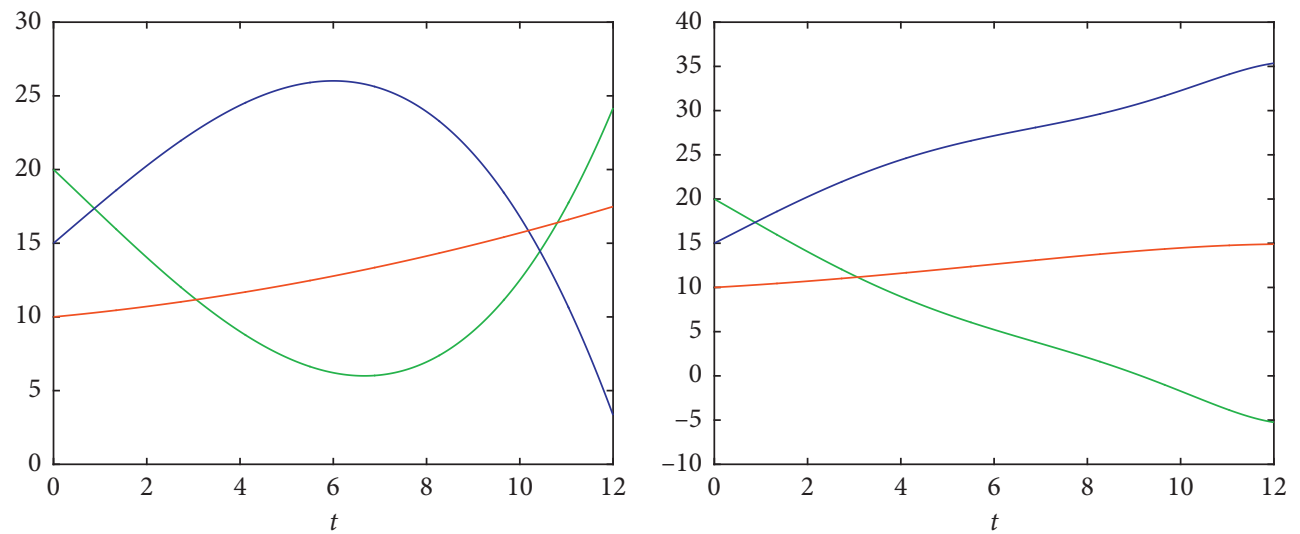

$\begin{array}{r}S(t) \\ -\quad I(t) \\ \hline\end{array}$

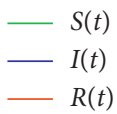

(a)

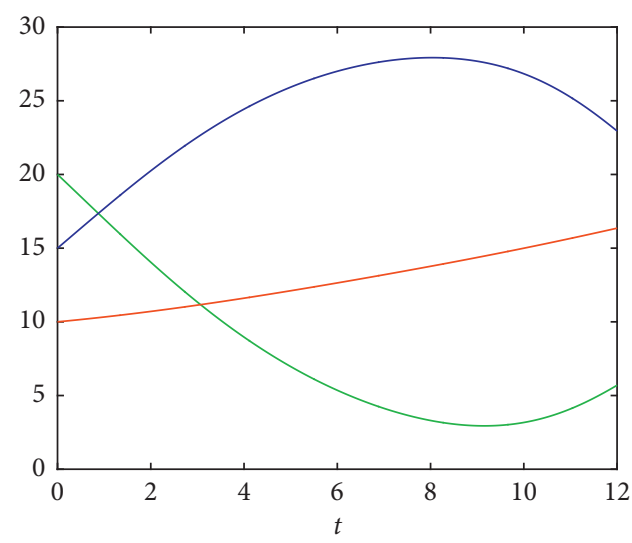

(b)

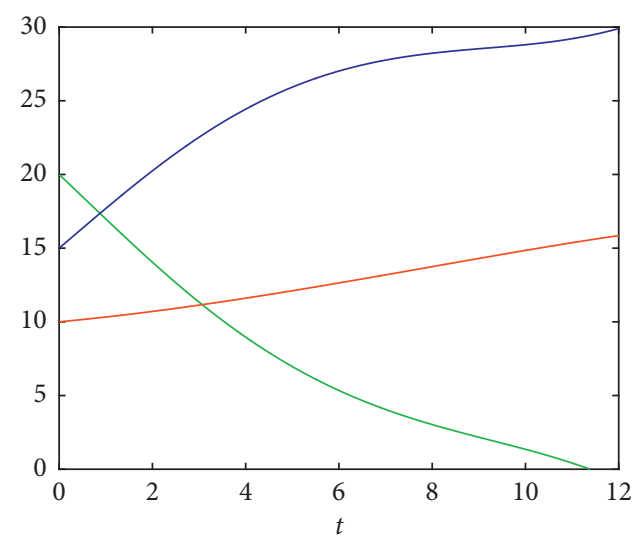
$-S(t)$
$-S(t)$
$-I(t)$
$-I(t)$
$-R(t)$
$-R(t)$

(c)

(d)

Figure 1: Three-, four-, five-, and six-term approximations for $S(t), I(t)$, and $R(t)$ versus time.
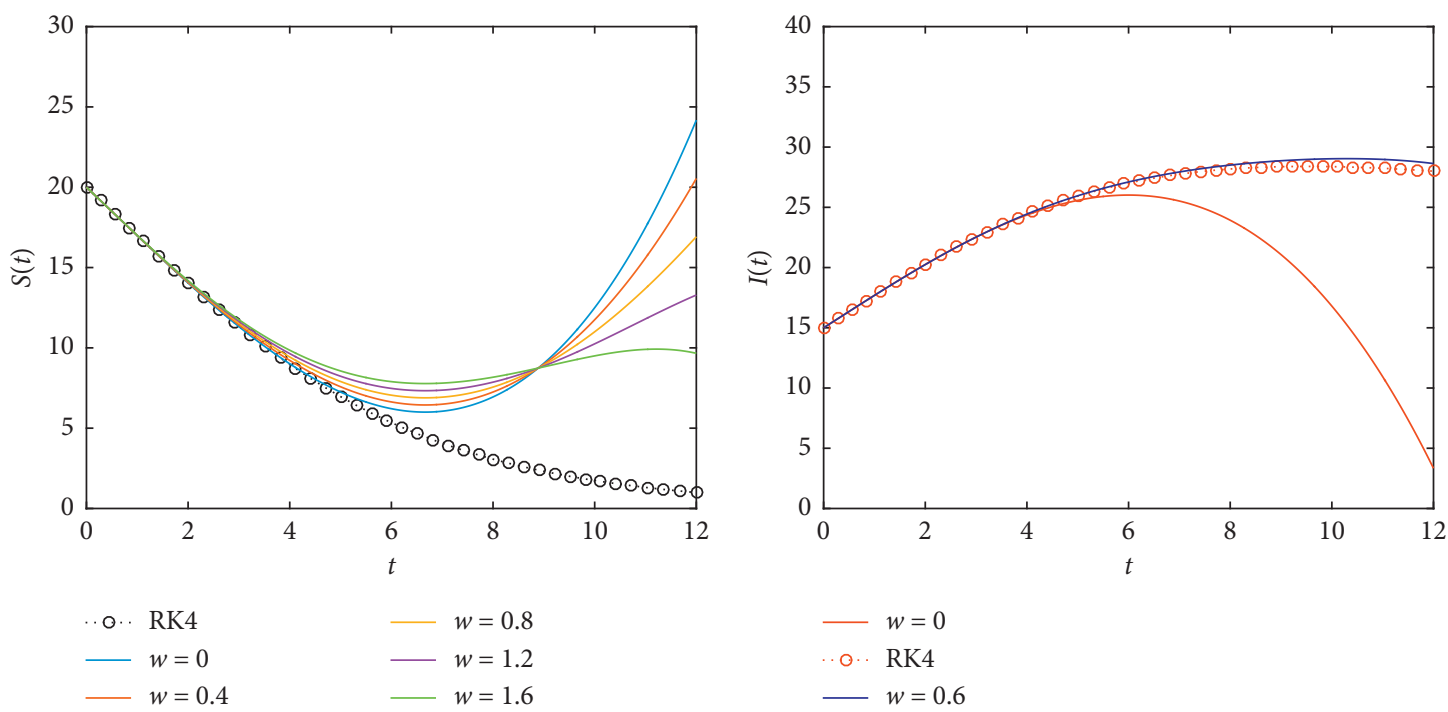

$w=0$

.. O.. RK4

(a)

(b)

Figure 2: Continued. 


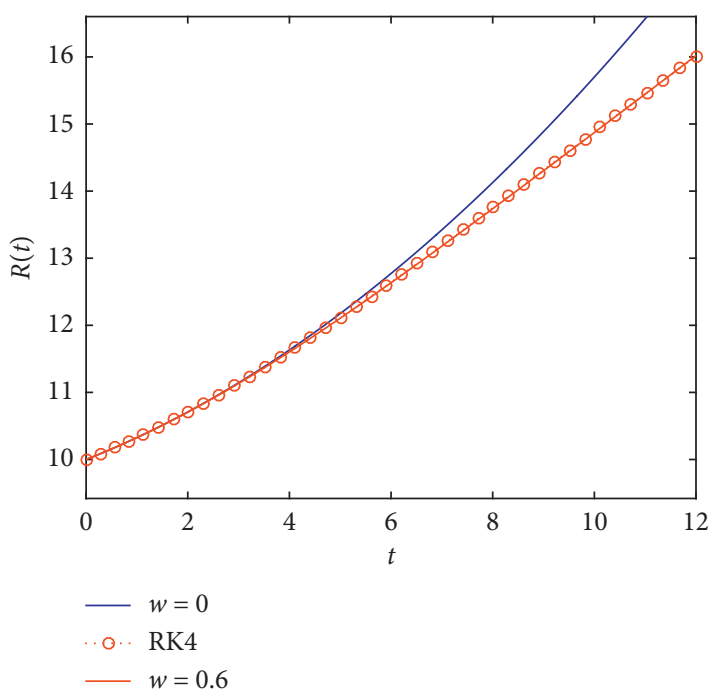

(c)

FIGURE 2: Comparison of three-term approximation SOR-like new iterative method, NIM, and RK4 for $S(t), I(t)$, and $R(t)$ versus time and $\omega$.

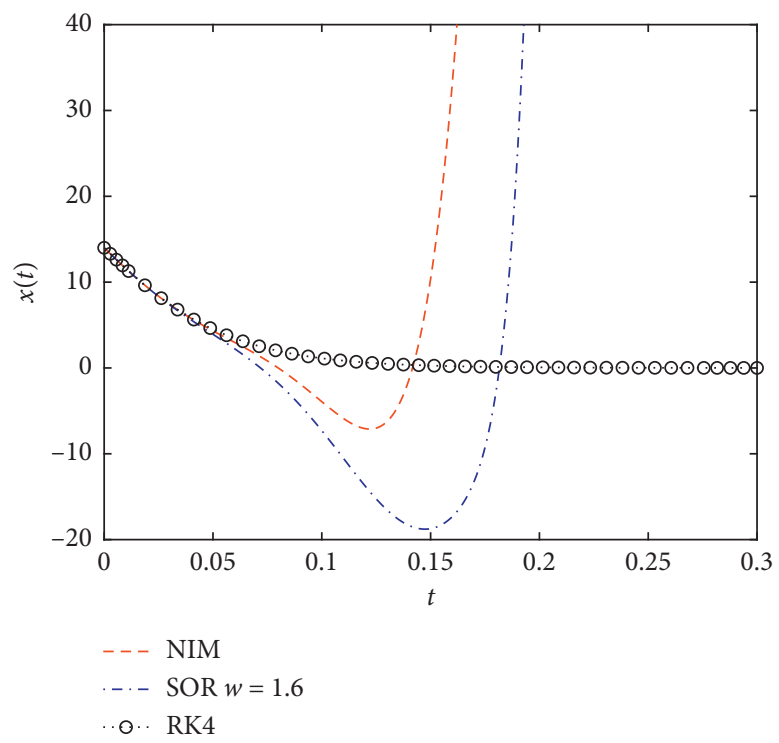

(a)

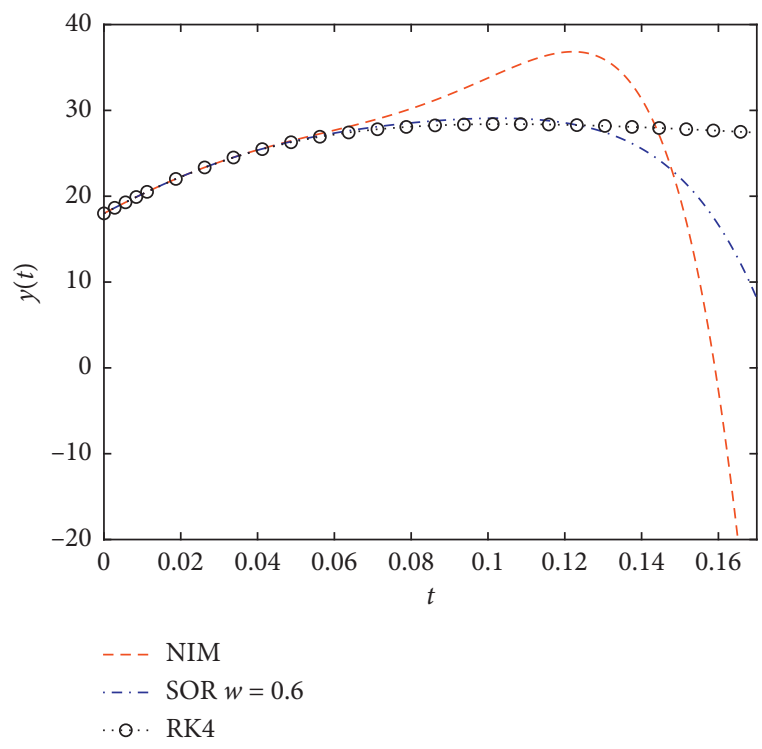

(b)

FIGURE 3: Case 1: comparison of three-term approximation SOR-like new iterative method, NIM, and RK4 for $x(t), y(t)$, and $\omega$.

A comparison between the results obtained by the SOR-like method and the NIM, ADM, and VIM in cases $1-2$ is completely the same for $\omega=0$ and will not be plotted (see $[2,3,7])$.

But when we vary the values of $\omega$, we clearly notice that our proposed method gives better results compared to the NIM method if we compare them to the Runge-Kutta (RK4) method. The following plots show the relations between the number of foxes and the rabbits versus time (Figures 3 and 4).
3.2.2. Comparison with the Multistage SOR-Like New Iterative Method and RK4. When solving the prey and predator problem, all the methods ADM, VIM, and NIM give the accurate results but in a small time interval $[0,0.16]$ (Figure 4 ). Once the time interval becomes wide (as example $[0,1]$ ), these methods fail as shown in Figure 3. To overcome this difficulty, we use the multistage SOR-like new iterative method and show that our proposed approach works well and gives accurate results compared to the order-four Runge-Kutta (RK4) method as illustrated in Figure 5. 


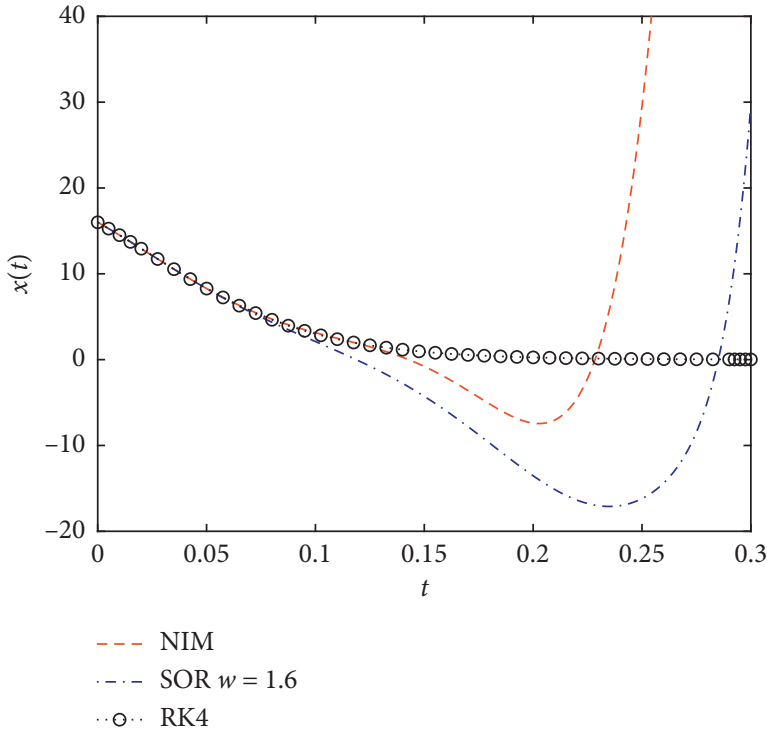

(a)

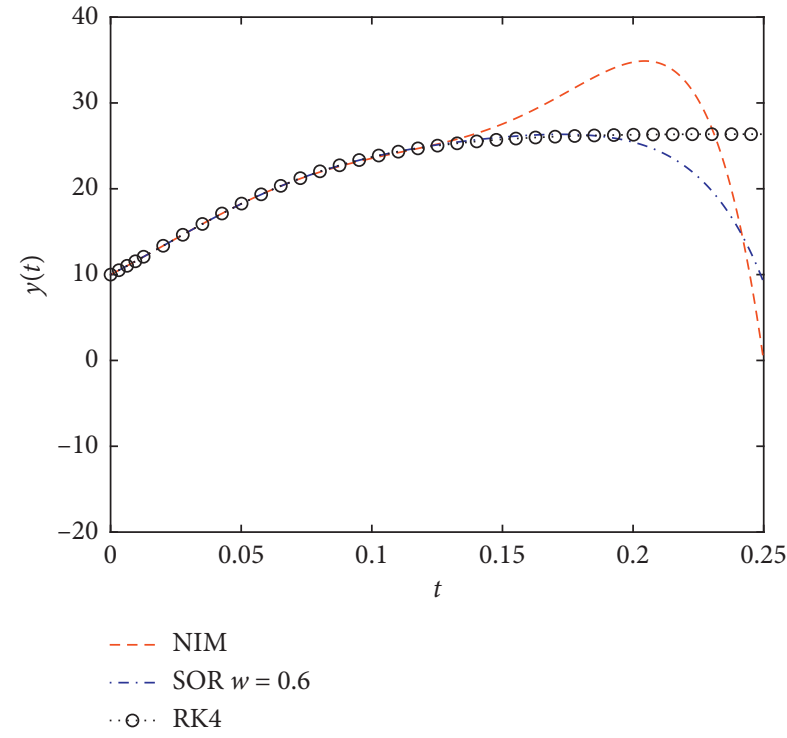

(b)

FIGURE 4: Case 2: comparison of three-term approximation SOR-like new iterative method, NIM, and RK4 for $x(t), y(t)$, and $\omega$.

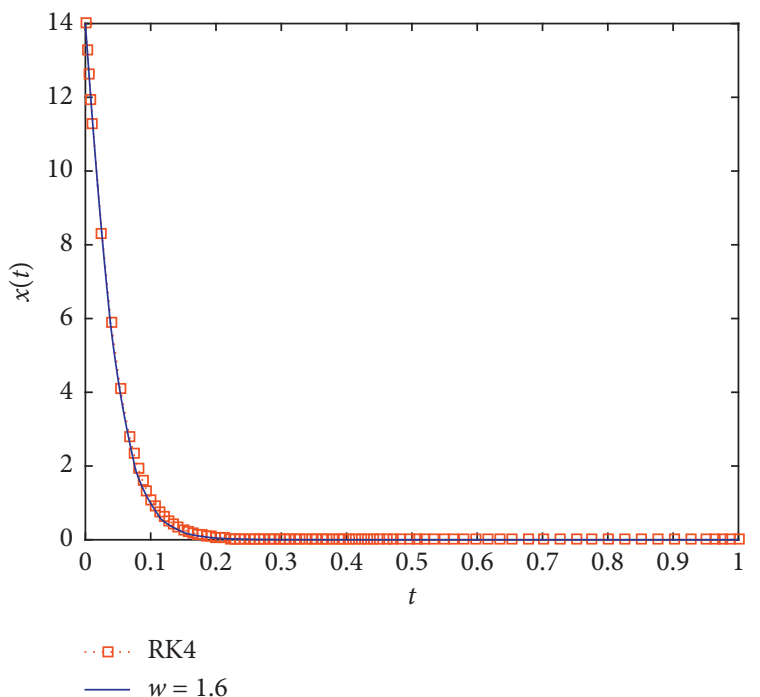

(a)

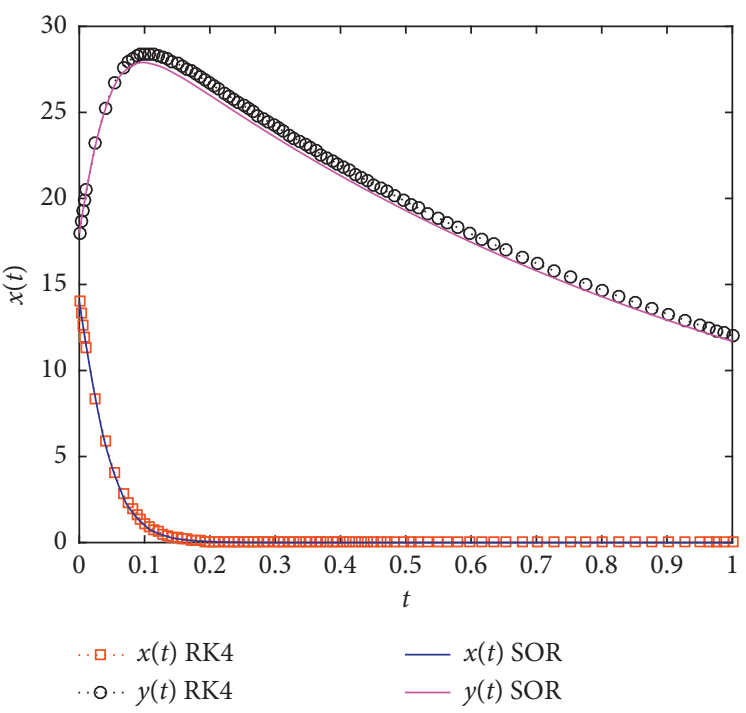

(b)

FIGURE 5: Comparison of multistage three term SOR-like and RK4 for $x(t), y(t)$, and $\omega=1.6$.

\section{Conclusion}

In this paper, we propose an improvement of a new iterative method called the SOR-like new iterative method to solve the nonlinear system of differential equations. The method introduces a relaxation parameter which in some cases makes it possible to find the well-known method. Compared to the nonlinear SOR-like method to solve the nonlinear algebraic system of equations, our method gives an accurate and fast approximation solution. We applied the proposed method to solve the epidemic model and the prey and predator problem successfully. When the time interval is wide, we introduce the multistage. The advantages of the proposed method are being derivative-free, simple to understand, and easy to implement and can be easily comprehended with only a basic knowledge of calculus. Compared to the Adomian decomposition method, the homotopy perturbation method, the variational iteration method, and the new iterative method, there is less computation needed. As perspectives, we will apply our method to solve the system of nonlinear stiff differential equations. For computations and plots, the MATLAB R2012 is used. 


\section{Data Availability}

All algorithms and data used to support the findings of this study are included within the article and available from the corresponding author upon request.

\section{Conflicts of Interest}

The authors declare that they have no conflicts of interest.

\section{References}

[1] W. O. Kermack, A. G. McKendrick, and G. T. Walker, "A contribution to the mathematical theory of epidemics," Proceedings of the Royal Society, vol. 115, pp. 700-721, 1927.

[2] J. Biazar, "Solution of the epidemic model by Adomian decomposition method," Applied Mathematics and Computation, vol. 173, no. 2, pp. 1101-1106, 2006.

[3] M. Rafei, H. Daniali, and D. D. Ganji, "Variational iteration method for solving the epidemic model and the prey and predator problem," Applied Mathematics and Computation, vol. 186, no. 2, pp. 1701-1709, 2007.

[4] Y. M. Rangkuti, S. Side, S. Side, and M. S. M. Noorani, "Numerical analytic solution of SIR model of dengue fever disease in South Sulawesi using homotopy perturbation method and variational iteration method," Journal of Mathematical and Fundamental Sciences, vol. 46, no. 1, pp. 91-105, 2014.

[5] J. Biazar and R. Montazeri, "A computational method for solution of the prey and predator problem," Applied Mathematics and Computation, vol. 163, no. 2, pp. 841-847, 2005.

[6] M. Rafei, H. Daniali, D. D. Ganji, and H. Pashaei, "Solution of the prey and predator problem by homotopy perturbation method," Applied Mathematics and Computation, vol. 188, no. 2, pp. 1419-1425, 2007.

[7] M. AL-Jawary, "A reliable iterative method for solving the epidemic model and the prey and predator problems," International Journal of Basic and Applied Sciences, vol. 3, no. 4, pp. 441-450, 2014.

[8] G. F. Simmons, Differential Equations with Applications and Historical Notes, McGraw-Hill, New York, NY, USA, 1972.

[9] M. S. H. Chowdhury, I. Hashim, and S. Mawa, "Solution of prey and predator problem by numeric-analytic technique," Communications in Nonlinear Science and Numerical Simulation, vol. 14, pp. 1008-1012, 2009.

[10] J.-H. He, "The homotopy perturbation method for nonlinear oscillators with discontinuities," Applied Mathematics and Computation, vol. 151, no. 1, pp. 287-292, 2004.

[11] J.-H. He, "Comparison of homotopy perturbation method and homotopy analysis method," Applied Mathematics and Computation, vol. 156, no. 2, pp. 527-539, 2004.

[12] G. Adomian and G. E. Adomian, "A global method for solution of complex systems," Mathematical Modelling, vol. 5, no. 4, pp. 251-263, 1984.

[13] G. Adomian and R. Rach, "On the solution of Algebraic equations by the decomposition method," Journal of Mathematical Analysis and Applications, vol. 105, no. 1, pp. 141166, 1985.

[14] J.-H. He, "Variational iteration method - a kind of non-linear analytical technique: some examples," International Journal of Non-linear Mechanics, vol. 34, no. 4, pp. 699-708, 1999.

[15] J. H. He, "Variational iteration method for autonomous ordinary differential systems," Applied Mathematics and Computation, vol. 114, no. 2-3, pp. 115-123, 2000.
[16] V. Daftardar-Gejji and H. Jafari, "An iterative method for solving nonlinear functional equations," Journal of Mathematical Analysis and Applications, vol. 316, no. 2, pp. 753-763, 2006.

[17] S. Bhalekar and V. Daftardar-Gejji, "New iterative method: application to partial differential equations," Applied Mathematics and Computation, vol. 203, no. 2, pp. 778-783, 2008.

[18] S. Bhalekar and V. Daftardar-Gejji, "Solving evolution equations using a new iterative method," Numerical Methods for Partial Differential Equations, vol. 26, pp. 906-916, 2010.

[19] V. Daftardar-Gejji and S. Bhalekar, "Solving fractional boundary value problems with Dirichlet boundary conditions using a new iterative method," Computers \& Mathematics with Applications, vol. 59, no. 5, pp. 1801-1809, 2010.

[20] V. Daftardar-Gejji and H. Jafari, "Convergence of the new iterative method," International Journal of Differential Equations, vol. 2011, Article ID 989065, 10 pages, 2011.

[21] S. Bhalekar and V. Daftardar-Gejji, "Solving a system of nonlinear functional equations using revised new iterative method," International Journal of Mathematical and Computational Sciences, vol. 6, no. 8, pp. 968-972, 2012.

[22] K. Ishihara, Y. Muroya, and T. Yamamoto, "On nonlinear SOR-like methods, II - convergence of the SOR-Newton method for mildly nonlinear equations," Japan Journal of Industrial and Applied Mathematics, vol. 14, no. 1, pp. 99-110, 1997. 